\title{
A cooperative multitasking model for networked sensors
}

\author{
Silvana Rossetto and Noemi Rodriguez \\ Departamento de Informática, PUC-Rio, Brazil \\ silvana,noemi@inf.puc-rio.br
}

\begin{abstract}
This paper proposes a concurrency model which integrates the asynchronous and event-driven nature of networked sensors with a more familiar programming style for the developer. We argue that coroutines can provide a basis for this integration and describe some details of its implementation, which was developed as an extension to the TinyOS operating system.
\end{abstract}

Keywords Sensor networks, TinyOS, programming models, event-driven model, cooperative multitasking, coroutines

Technical Areas Programming abstractions for sensor networks, operating system and middleware support

\section{Introduction}

Recent advances in low-power microcontrollers and wireless communication have enabled multifunctional tiny devices which can be densely deployed throughout a physical space to sense, process and communicate required data. Similarly to other embedded systems, networked sensors must respond to different stimuli, including physical events and messages from other devices. Besides, networked sensors are highly unstructured and dynamic environments, in which there are no well-behaved communication patterns. As a consequence, computing models for such systems are typically event-driven and asynchronous [4, 6].

In their simplest form, event-driven models encompass a set of independent activities or services, each of them with a single execution line that handles each incoming event at a time. This leads to a state-machine programming model, in which the arrival of a new event can trigger a transition that depends on the current state. Although conceptually simple, this is not the classical way in which programmers are used to thinking. When an operation cannot complete immediately, it must be split across one or more invocations of the event handler, forcing the programmer to code "continuations" within his handlers. Between event handler invocations, new events may occur, triggering new logical execution lines. The developer must, thus, construct his program as a state machine and face issues such as maintaining consistent state across multiple logical tasks. This can impose high programming costs.

In this paper, we propose a programming model which combines the asynchronous basis of event-driven systems with a more classical programming interface for the developer. The central idea is to investigate a computing model that takes into account the limited resources of sensor devices while supporting higherlevel programming paradigms able to reduce the cost of developing sensing applications. We use cooperative multitasking to support lightweight concurrency without forcing the programmer to manually build continuations within an application.

The traditional approach to dealing with concurrent execution lines is to use multithreading. However, multithreading is typically preemptive, that is, the programmer has no control over the moment at which control switches occur. This imposes context switching overhead and the need to deal with race conditions and deadlocks. One alternative is to use coroutines [13, 7], a programming language construct that enables a process to maintain distinct execution lines and explicitly alternate the control among them. Thus, distinct parts of an application can suspend only it is impossible for them to continue immediately and be resumed later, when their processing requirements become available. That is, coroutines are a collaborative and nonpreemptive type of multitasking. This aspect is fundamental in making coroutines suitable for networked sensors: context switching occurs only it is necessary (reducing the computing cost associated to it) and most concurrency issues (such as race conditions) can be implicitly avoided.

To experiment with the coroutine abstraction for networked sensors, we have integrated a coroutinebased concurrency model into TinyOS [11], a wellknown operating system for networked sensors. Basi- 
cally, we have implemented a simple API and a coroutine scheduler that allow us to transform two-phase operations (typically specified by means of the pair command/event in a TinyOS interface), into one operation that is appropriately suspended and resumed, allowing the programmer to maintain a sequential view of his application. In this paper, we discuss the implementation of this model and the gain of using it in developing a sensing application, and evaluate the associated computing cost.

The rest of this paper is organized as follows. Section 2 describes TinyOS. Section 3 presents the proposed concurrency model and discusses how we have integrated this model into TinyOS. Section 4 evaluates the cost of using coroutines in a sensing application. Finally, in Section 5, we include some final remarks.

\section{Systems for networked sensors}

Networked sensors consist of potentially thousands of tiny, low-power nodes, each of which execute concurrent, reactive programs that must operate with severe memory and power constraints. Information must be simultaneously captured from sensors, manipulated, and streamed onto a network. Moreover, nodes must deal with events that require real-time responses. An example is message arrival. Typically communication is radio-based. The radio is an asynchronous input/output device that contains no buffer, so each bit must be serviced by the node as soon as it becomes available. Systems for networked sensors thus present some special requirements. First, software solutions must make efficient use of processor and memory while enabling low power consumption. Second, it is necessary to maintain a number of concurrent flows and juggle numerous outstanding events.

TinyOS [11] is the current state of the art in operating systems for sensor network research. It implements a component-based model based on split-phase interfaces, asynchronous events and deferred computation. Hardware interruptions trigger immediate execution of event handlers. Tasks are the main unit of execution, and each task executes to completion, except that it may be interrupted by a hardware event (there is no concurrency among tasks). In the next section we describe some important design aspects of TinyOS.

\subsection{The TinyOS design}

TinyOS design is based on three programming constructs: commands, events, and tasks. Both commands and events are intended to perform small amounts of work. Commands are used to request services and com- plete immediately. Typically, a command handler deposits request parameters and conditionally posts a task for later execution. Events are signaled to indicate service (command) completion or hardware events. The lowest-level components have handlers connected directly to hardware interrupts, which can be external interrupts, timer events or counter events. An event handler can deposit information in the component's environment, post tasks, signal higher level events or call commands. Tasks are a form of deferred procedure call that allows postponing processing. They are atomic with respect to each other and run to completion, but can be preempted by interrupts (hardware events). Tasks allow concurrency within each component since they execute asynchronously with respect to events. To ensure low task execution latency, individual tasks are expected to be short, i.e, lengthy operations should be spread across multiple tasks. Posted tasks are executed by the TinyOS scheduler when the processor is idle.

TinyOS highlights separation of construction and composition. There are two different programming constructors: module, that is used to provide code; and configuration that is used to wire components together. The TinyOS component behavior is specified in terms of interfaces that can be provided or used by the component. Interfaces specify a multi-function interaction channel between two components, the provider and the user. The interface provider must implement a set of named functions called commands, and the interface user must implement the set of named functions, called events, that can be signaled upon completion of the commands it uses.

A C-like language called nesC [10] was specially designed to provide the event-driven concurrency model used by TinyOS. In order to avoid data races which can occur due to concurrent updates to shared state - nesC offers two options: either to implement all of the critical code inside tasks or to use atomic sections to update the shared state. Atomic sections are small code sequences that nesC ensures will run atomically by disabling and enabling interrupts.

\section{A new concurrency model for networked sensors}

Although it deals well with the main constraints of networked sensors, the programming model designed by TinyOS is not easy to use. Its multitasking engine maintains a two-level scheduling structure that is similar to a finite state machine model, which can be difficult to program. To develop a simple sensing application in nesC, the programmer must typically control shared memory access and partition basic requests into 


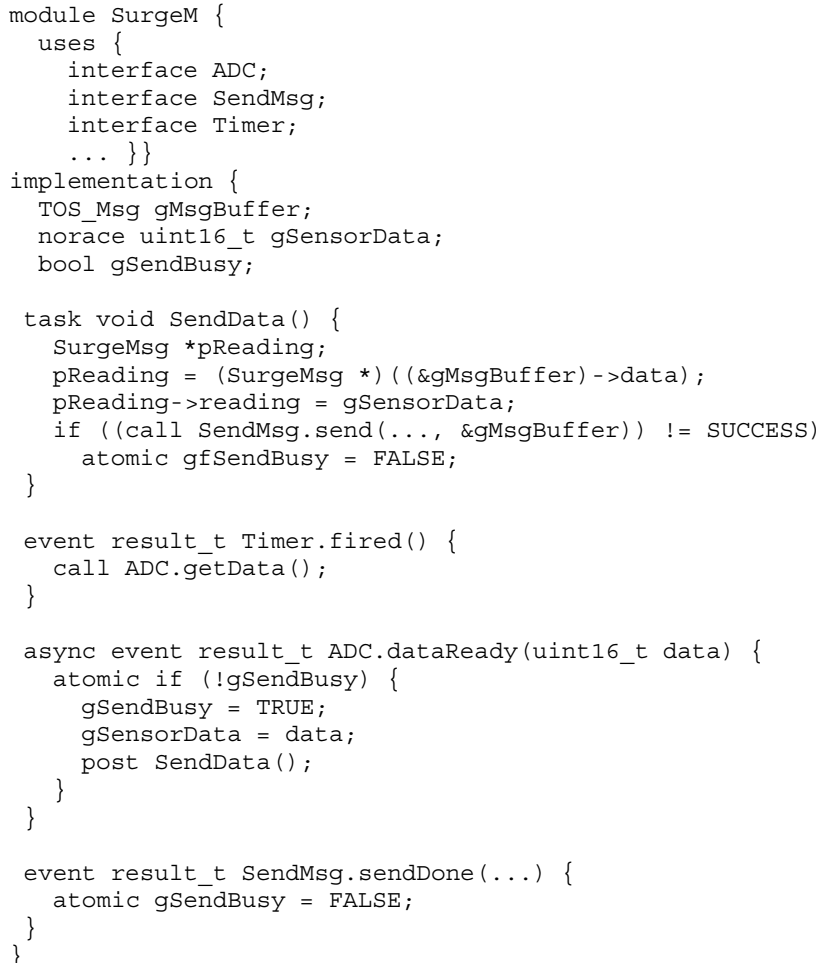

Figure 1. Core logic of the Surge application.

two-phase operations.

As an example, Figure 1 shows the main code of Surge, a nesC application in which nodes in the network take light readings and forward them to a base station. Each time the Timer. fired event is signaled, the $A D C$.getData command is called to get a new sensor value. When the sensor value is available, the hardware signals the ADC. dataReady event. As gSendBusy is accessed in a hardware event handler, its use must be protected by one atomic statement. The handler for this event finishes by posting the SendData task, which sends the value to the base station.

In this code, the main task (to take data readings and forward them) was broken into four distinct pieces of code, since sensor value reading is a typical splitphase operation. The ADC interface defines the command getData to request a reading; and the event dataReady to signal the sensor value is available.

Our goal is to provide a more intuitive programming abstraction to the developer. A classical and more convenient way to get data readings would be to call just one simple command and get the sensor value as the return value to this request. In our proposal, we encapsulate the two phases of a typical request/answer in a single request, enabling the programmer to struc- ture his application as sequential code instead of as a state machine. Figure 2 illustrates how the Surge code is simplified with this approach. In the original version, Timer. fired issues the ADC.getData command, and $A D C$. dataReady handles the event signaled by this command. In the new code, in Figure 2, operation newSendData contains both the invocation of $A D C$. getData and the handling of its result, eliminating the split-phase behavior.

We achieve this goal by employing coroutines. The possibilities brought by coroutines are in many ways similar to those offered by multithreading, but there are important differences. Multithreading [5] is a notion widely explored in order to enable processes to have distinct execution lines called threads. The developer defines the distinct threads of execution and the operating system, or an appropriate library, is responsible for relocating the processor control among threads. Coroutines allow the programmer to suspend an execution line to wait for some system event and restore this execution later, but context changes are explicit, avoiding race conditions. On the other hand, the programmer is responsible for control transfers.

In this work, we use coroutines to allow a networked sensor application to respond to system events continuously, modeling each split-phase operation as a new coroutine, and transferring control back to the main loop when it needs to wait for hardware events to continue. Control transference is encapsulated in blocking operations, so they will only occurs when it is necessary and the programmer need not explicitly deal with transfers.

We propose changing interfaces like ADC into new interfaces in which only commands are offered: blocking operations are transformed into split-phase calls; the context of execution is preserved; and yet the application is able to respond to other system events or deferred tasks while the operation is not able to continue. In order to support this scenario, the following three steps were necessary: (1) building a basic API with coroutine operations for sensors; (2) implementing a coroutine scheduler to resume coroutines automatically; (3) defining a step-by-step procedure to construct proxy interfaces, redefining TinyOS two-phase operations as one-phase operations.

\subsection{Coroutine operations for sensors}

Our first step was to implement support for the coroutine construct in TinyOS. A coroutine is represented by a code address and has its own stack. There are different syntactic and semantic ways to support coroutines [7]. We support asymmetrical coroutines, al- 


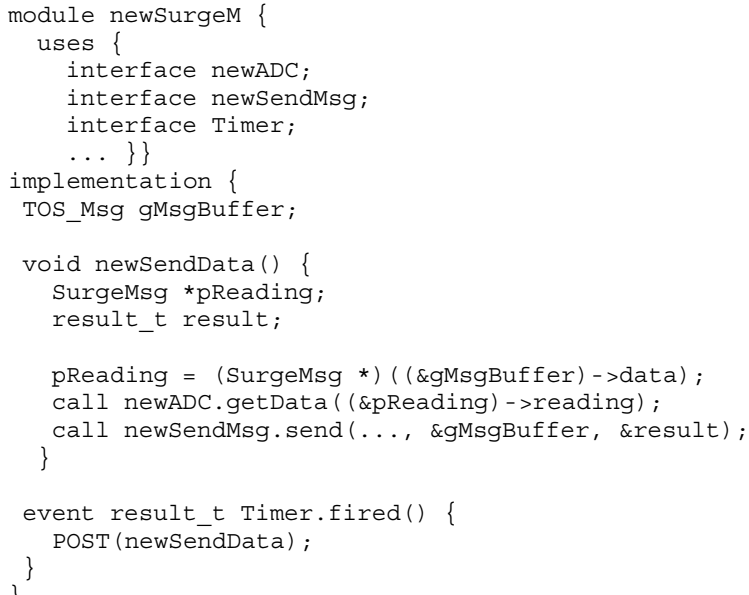

Figure 2. New core logic of the Surge application.

lowing arbitrary functions, independently written, to be invoked as coroutines. For this, we implemented a set of operations that allocate storage for a coroutine stack, associate a function to a coroutine storage area, transfer control to a coroutine, and yield control from a coroutine.

A key issue related to coroutine implementation is how to pass parameters between two coroutines. It is difficult because we typically use the stack to pass parameters and each coroutine uses a different stack. Moreover, a typical coroutine can have several entry points (immediately after each transfer operation). To maintain our implementation simple, we use (scope restricted) global variables to transfer data.

Implementation We implemented coroutines for the microcontroller ATmega128L [1] (from Atmel-AVR family [2]). It has $128 \mathrm{~KB}$ of flash for program memory and $4 \mathrm{~KB}$ of SRAM for data memory. The coroutines stacks are allocated on the heap.

One simple way to implement coroutines is by using the setjmp and longjmp functions [9]. The setjmp function saves the current execution context (including the stack pointer) into a pre-defined data structure to be passed later as argument to the longj mp function. The longjmp function, in its turn, resumes the execution context previously saved by set jmp.

The AVR Libc [3] library (a subset of ANSI-C library for AVR microcontrollers) implements setjmp and longjmp functions and defines a specific data structure, called jmp_buf, to be used by these functions. To implement the coroutine construct, we do a setjmp to save the initial state in the jmp_buf struc- ture and then manually adjust the program counter and the stack pointer fields.

\subsection{Coroutine scheduler}

TinyOS provides a queue for all tasks and a scheduler to execute these tasks using a FIFO policy. We have extended the TinyOS task scheduler to include a coroutine queue (with pre-allocated space) and a coroutine scheduler that resumes coroutines that are ready. The TinyOS scheduler is implemented as a set of $\mathrm{C}$ functions in the file "sched.c". Since we are using TinyOS 1.x, we have changed this file directly (a future step is to update this implementation to TinyOS 2.0 by designing an appropriate scheduler component).

A TOS_post_coro function (similar to TOS_post) was implemented. Basically, this function receives a function as argument and associates it to an available coroutine. The coroutine is then marked as "ready" and can be resumed by the scheduler. We have changed the TinyOS main loop to call a function (similar to TOSH_run_next_task) that checks and resumes all ready coroutines.

In order to offer access to coroutine services from the component level, a set of functions are exported by file "sched.c", which includes:

- bool POST (procedure_t proc): schedules a procedure to be executed as a coroutine;

- uint8_t GETID (): returns the current coroutine;

- void SUSPEND(): transfers control execution back to the main coroutine;

- void RESTORE (uint8_t id): informs the scheduler that the given coroutine is ready to be resumed.

\subsection{Proxy for current TinyOS interfaces}

Using the new TinyOS scheduler and the set of functions exported by it, we can rewrite typical TinyOS interfaces, encapsulating in their implementation the transfer of control between coroutines. As a result, the programmer that uses such an interface in his application will be provided with the sequential view we wanted. Figure 3 presents, as an example of this, the interface proxy we have implemented to replace the ADC interface.

In the new version of the ADC interface, the newADC.getData command calls the original command ADC.getData and suspends the current execution line by calling SUSPEND. At this point the execution control goes back to the main loop, so the application is 


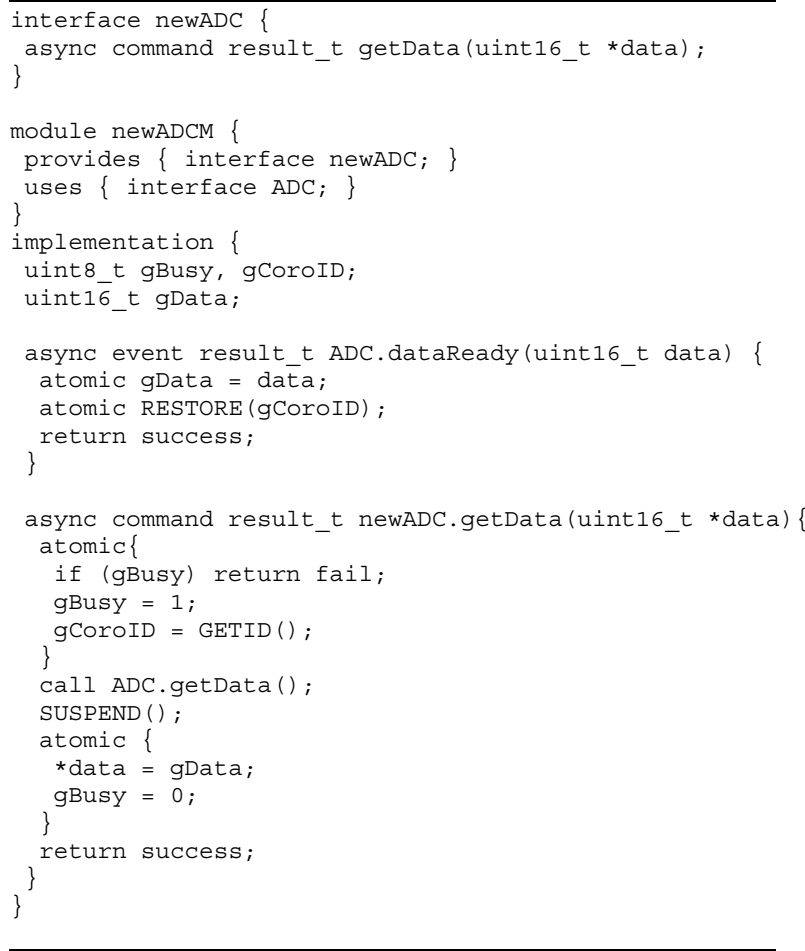

Figure 3. Proxy for ADC interface.

able to handle other events or tasks. The coroutine identifier is stored in the gCoroID variable to be used later, when this coroutine becomes ready to continue.

When the requested value is available, the $A D C$.dataReady event is signaled. This means the previous suspended coroutine can continue its execution. The coroutine scheduler is notified by calling the RESTORE function. The next time this coroutine is resumed, the execution will continue after the SUSPEND () statement.

Besides appropriately suspending and resuming the getData task, the proxy for the ADC interface encapsulates all shared memory access. So, the programmer need not worry with atomic accesses to the sensed data. We have used the same step-by-step procedure to construct a proxy for the SendMsg interface. Using these proxies the programmer can write the simplified version of the Surge application, presented in Figure 2.

\section{Evaluation}

In order to evaluate the overhead added by coroutines, we compare the simulated behavior of the Surge code presented in Figure 1 and Figure 2, at the machine code level. Because we are using setjmp/longjmp functions to implement coroutines, it is necessary to use a program that allows for emulating, in an instruction by instruction manner, the operation of individual sensor nodes. For that, we adopted ATEMU [14], a fine grained sensor network simulator. Like TOSSIM [12], (a simulator specially designed for TinyOS programs), ATEMU simulates sensor networks in which the nodes are AVR microcontrollers [2] and runs the microcontroller program, rather than models of the software. However, TOSSIM uses a few system libraries - instead of AVR Libc library - when compiling nesC code into a binary for the development workstation. Among these libraries is the one that supports setjmp/longjmp functions.

Figure 4 shows the results of simulations we have conducted. We used the ATmega128L Emulator (version 0.4) provided by ATEMU. In the first case, we simulated the core Surge code (presented in Figure 1) compiled with TinyOS 1.x. In the second case, we replaced the original "sched.c" file of TinyOS 1.x by our new implementation, which adds an initialization step to allocate memory space for coroutines and a coroutine scheduler (our goal in this case was to measure the overhead imposed by the new scheduler even if we don't use coroutines). Finally, in the third case, we simulated the new version of the Surge code (presented in Figure 2). We are interested in comparing the number of clock cycles needed to start the application and to take the first sensor reading in each case.

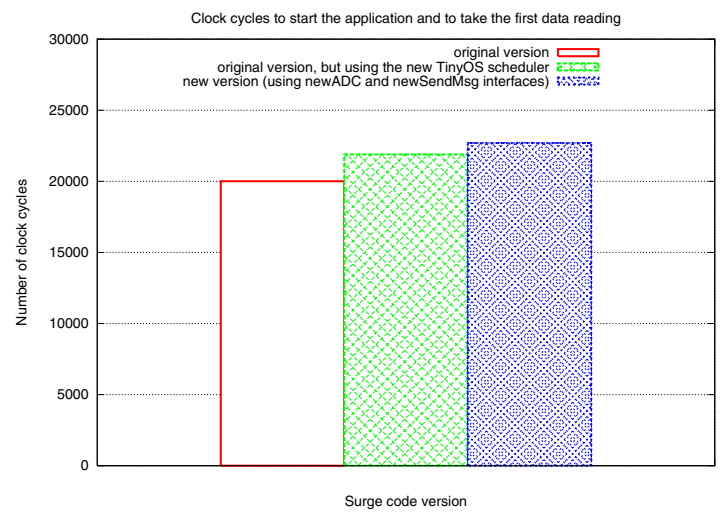

Figure 4. Clock cycle overhead added by coroutines.

The first results are encouraging. The overhead added by the new scheduler is approximately $9.4 \%$ and the further overhead added by using coroutines is about $3.6 \%$ (the total overhead in the third case is $13.4 \%$ ). With respect to code size, basically we have just increased the size of the "sched.c" file (which contains all coroutine operations and the coroutine scheduler) in approximately $60 \%$. These are the results we obtained 
with our initial implementation, which we believe we can still improve.

\section{Final Remarks}

Software architectures for networked sensors are typically concurrent and event driven. However, eventtriggered programming models are not natural for programmers: applications have to be written as explicit state machines, which are hard to understand and maintain. In this work, we proposed a coroutine-based concurrency model for networked sensors. By using the coroutine abstraction, we can build a sequential view for the programmer, without losing the event-based and asynchronous characteristics required by the networked sensors. Coroutines are a lightweight construct and seem to match well the constraints of sensor networks.

The work presented in [8] on Protothreads also proposes a programming abstraction which intends to reduce the complexity of high-level programs in eventtriggered sensor nodes systems. Unlike coroutines, protothreads implement a type of continuation (called local continuation) that does not require its own stack: all protothreads run on the same stack and context switching is done by stack rewinding. The main limitation of protothreads is that variables with function-local scope are not automatically saved across blocking operations because the stack is rewound at every blocking statement. In our system, each coroutine has its own stack, and thus maintains its local variables across control transfers. Besides, since protothreads are implemented using the $C$ switch statement, programs cannot utilize switch statements together with protothreads.

Welsh and Mainland [16] propose abstract regions to abstract interaction details between nodes in a sensor network. To simplify the programming task using abstract regions, the authors implemented a synchronous programming interface for TinyOS based on "lightweight threads". The system maintains two execution flows: a main flow, which is event-driven and cannot block; and an application flow, which can invoke blocked operations. The same stack is shared by these two flows of execution. Using this structure, the application can block while the system remains eventdriven. In our proposal, the main flow is event-driven and the application can be divided into more than one control flow, each one with its own stack of execution.

This work is part of a project in which we study applications of cooperative multitasking. In a previous paper [15], we discussed how coroutines can be used to couple the advantages of asynchronous communication with the use of the well-known remote procedure call abstraction in geographically distributed systems.
Acknowledgments Work partly supported by CNPq: a Brazilian government research agency.

\section{References}

[1] Atmega128(1) summary. http://www.atmel.com/.

[2] Atmel corporation. http://www.atmel.com/.

[3] Avr libc. http://www.nongnu.org/avr-libc/.

[4] I. F. Akyildiz, W. Su, Y. Sankarasubramaniam, and E. Cayirci. Wireless sensor networks: A survey. Comput. Networks, 38(4):393-422, 2002.

[5] A. Birrell. An Introduction to Programming with Threads, pages 88-118. Prentice Hall, 1991.

[6] D. Culler, D. Estrin, and M. Srivastava. Overview of sensor networks. Computer, 37(8):41-49, Aug 2004.

[7] A. L. de Moura, N. Rodriguez, and R. Ierusalimschy. Coroutines in Lua. Journal of Universal Computer Science, 10(7):910-925, 2004.

[8] A. Dunkels, O. Schmidt, and T. Voigt. Using Protothreads for Sensor Node Programming. In Proceedings of the Workshop on Real-World Wireless Sensor Networks, Stockholm, Sweden, June 2005.

[9] R. Engelschall. Portable multithreading: The signal stack trick for user-space thread creation. In Usenix Annual Technical Conference, pages 239-250, San Diego, CA, USA, 2000.

[10] D. Gay, P. Levis, R. von Behren, M. Welsh, E. Brewer, and D. Culler. The NesC language: A holistic approach to networked embedded systems. In Conference on Programming Language Design and Implementation, pages 1-11, New York, USA, 2003. ACM Press.

[11] J. Hill, R. Szewczyk, A. Woo, S. Hollar, D. Culler, and K. Pister. System architecture directions for networked sensors. In 9th International Conference on Architectural Support for Programming Languages and Operating Systems (ASPLOS-IX), pages 93-104, New York, NY, USA, 2000. ACM Press.

[12] P. Levis, N. Lee, M. Welsh, and D. Culler. TOSSIM: Accurate and scalable simulation of entire TinyOS applications. In 1st ACM Conference on Embedded Networked Sensor Systems, pages 126-137. ACM Press, 2003.

[13] C. D. Marlin. Coroutines: A programming methodology, a language design and an implementation. Lecture Notes in Computer Science, 95, 1980.

[14] J. Polley, D. Blazakis, J. McGee, D. Rusk, and J. Baras. Atemu: A fine-grained sensor network simulator. In Sensor and Ad Hoc Communications and Networks, pages 145-152, 2004.

[15] N. Rodriguez and S. Rossetto. Integrating remote invocations with asynchronism and cooperative multitasking. In Third International Workshop on High-level Parallel Programming and Applications (HLPP'05), Warwick, Inglaterra, 2005.

[16] M. Welsh and G. Mainland. Programming sensor networks with abstract regions. In USENIX/ACM Symposium on Network Systems Design and Implementation, 2004. 